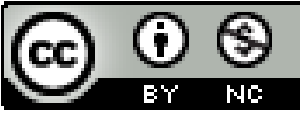

\title{
A ABORDAGEM CARROLLIANA A PARADOXOS
}

\author{
The carrollian approach to paradoxes
}

\author{
John Lennon Lindemann \\ UFSM \\ Frank Thomas Sautter \\ UFSM
}

\begin{abstract}
Resumo: O objetivo deste trabalho é apresentar a versão carrolliana de dois paradoxos clássicos (Pseudomenos e Antistrephon) e um original (On The Way to the Barber-Shop), acompanhadas da reconstrução e exame do tratamento lógico oferecido por Carroll e de como tais paradoxos foram tratados por outros autores.
\end{abstract}

Palavras-chave: Lógica; Lewis Carroll; Paradoxos; Método por Subscritos.

Abstract: The purpose of this paper is to present the Carrollian version of two classical paradoxes (Pseudomenos and Antistrephon) and an original (On The Way to the Barber Shop), with the reconstruction and examination of the logical treatment offered by Carroll and how such paradoxes were treated by other authors.

Keywords: Logic; Lewis Carroll; Paradoxes; Method of Subscripts.

\section{Introdução}

Lewis Carroll, pseudônimo de Charles Lutwidge Dodgson (1832-1898), é amplamente reconhecido como o principal responsável pela popularização da lógica em ambientes não acadêmicos durante o século XIX (THE JOY OF LOGIC, 2013) ${ }^{1}$, não apenas pela qualidade didática de seus trabalhos em lógica, mas também pelas suas obras literárias (MONTOITO, 2011, p. 9)², que estimulam o raciocínio lógico dos leitores. Seu legado não se limita à divulgação da lógica, tendo feito contribuições originais na área, como o seu método de árvores, um "método de decisão para sorites que pode ser considerado o precursor das atuais árvores de refutação" (SAUTTER, 2004, p. $91)^{3}$.

Carroll publicou apenas dois trabalhos teóricos sobre lógica: “The Game of Logic"(1887)4, um manual de lógica onde apresenta o seu método diagramático para a resolução de silogismos como um jogo de peças, e "Symbolic Logic: Part I - Elementary" $(1896)^{5}$, uma introdução à lógica, ao seu método diagramático e ao seu método por subscritos com uso limitado à silogística.

${ }^{1}$ THE JOY OF LOGIC. Direção: Catherine Gale. 59 min, [S.I.]: BBC, 2013.

http://www.dailymotion.com/video/xla6ogd the-joy-of-logic tech (último acesso em 03/09/2017).

${ }^{2}$ MONTOITO, R. Chá com Lewis Carroll: a matemática por trás da literatura. Jundiaí: Paco Editorial,

2011, p. 9.

3SAUTTER, F. "Lewis Carroll e a pré-história das árvores de refutação". In. SAUTTER, F. T. \&

FEITOSA, H. de A. (Eds.). Lógica: teoria e reflexões. Coleção CLE. Campinas, 2004, p. 91.

${ }^{4}$ CARROLL, L. The Game of Logic. 2 ed. London: MacMillan and Co., 1887, 96 p.

${ }^{5}$ CARROLL, L. Symbolic Logic: Part 1 -Elementary. Londres: MacMillan and Co., 1896, 188 p. 
Em 1977, após minucioso trabalho de compilação dos manuscritos de Carroll realizado por William Warren Bartley III (1934-1990), é publicada uma nova edição de "Symbolic Logic: Part I" (1986, p. 59-228) ${ }^{6}$ em conjunto com a publicação inédita da obra póstuma chamada "Symbolic Logic: Part II Advanced" (1986, p. 229-514)7 , na qual Carroll apresenta temas avançados em lógica, incluindo o seu método de árvores.

No "Book XXI" de "Symbolic Logic: Part II" (1986, p. 423-475) ${ }^{8}$, Carroll oferece tratamento lógico para alguns paradoxos clássicos e outros originais, chamados de "quebra-cabeças" pelo autor.

"Os paradoxos foram excepcionalmente importantes para os matemáticos e também para os algebristas da lógica no final do século XIX", inclusive o trabalho de Frege foi "motivado pela crise nos fundamentos da matemática gerada pela descoberta de paradoxos" (LINDEMANN, 2017, p. 23) $)^{9}$.

O objetivo do presente trabalho é apresentar três paradoxos da obra de Carroll, dois clássicos, a saber, "Pseudomenos" (1986, p. 425) ${ }^{10}$ e "Antistrephon" (1986, p. 426) ${ }^{11}$, e um original, "On the Way to the Barbershop" (1986, p. 428-431) ${ }^{12}$, acompanhados da reconstrução e do exame do tratamento lógico dado por Carroll e de como tais paradoxos foram tratados por outros autores.

Antes da exposição dos paradoxos, faz-se mister uma breve apresentação do método por subscritos, necessária para a compreensão do tratamento lógico que Carroll oferece aos paradoxos e útil para que seu trabalho seja situado no contexto histórico de desenvolvimento da lógica que Ihe é adequado.

\section{O Método por Subscritos}

Antes do reconhecimento e da popularização das inovações contidas na obra "Begriffsschrift" (1879)13 ${ }^{13}$, de Gottlob Frege (1848-1925), que "constitui desde então a base da lógica moderna" (KENNY, 1999, p. 438) ${ }^{14}$, não havia um instrumental lógico com o poder do cálculo de predicados, capaz de reconhecer a validade de silogismos e de argumentos não silogísticos com a mesma interpretação restrita do sistema de signos.

As obras de Carroll se situam no período da álgebra da lógica (BARTLEY III, 1986, p. 19) ${ }^{15}$, onde a lógica aristotélica perde sua hegemonia e argumentos proposicionais já não são ignorados, mas os lógicos ainda não desfrutam das inovações de Frege $(1879)^{16}$. Uma certa plasticidade foi recorrente no trabalho dos lógicos do período, onde a mesma notação utilizada para a lógica de termos era passível de uma leitura alternativa, usada para a lógica de proposições.

\footnotetext{
${ }^{6}$ CARROLL, L. Symbolic Logic: Lewis Carroll's. 6 ed. Rev., ampl. e atual. New York: Clarkson Potter, 1986, $514 \mathrm{p}$.

${ }^{7}$ lbid.

8Ibid.

${ }^{9}$ LINDEMANN, J. L. A Lógica de Lewis Carroll. Dissertação (Mestrado em Filosofia) - Programa de Pós-graduação em Filosofia, Universidade Federal de Santa Maria, 2017, p. 26.

${ }^{10}$ CARROLL, L. Symbolic Logic: Lewis Carroll's. 6 ed. Rev., ampl. e atual. New York: Clarkson Potter, $1986,514 \mathrm{p}$.

${ }^{11}$ Ibid.

${ }^{12}$ Ibid.

${ }^{13}$ FREGE, G. Begriffsschrift: eine der arithmetischen nachgebildete Formelsprache des reinen Denkens. Halle, Alemanha: Louis Nebert, 1879, 89 p.

${ }^{14}$ KENNY, A. História Concisa da Filosofia Ocidental. Tradução de Desidério Murcho,Fernando Martinho, Maria José Figueiredo, Pedro Santos e Rui Cabral. Lisboa: Temas e Debates Actividades Editoriais, 1999, p. 438.

${ }^{15}$ BARTLEY III. W. W. “Annotations”. In: CARROLL, L. Symbolic Logic: Lewis Carroll's. 6. ed. Rev., ampl. e atual. New York: Clarkson Potter, 1986, 514 p.

${ }^{16}$ FREGE, G. Begriffsschrift: eine der arithmetischen nachgebildete Formelsprache des reinen Denkens. Halle, Alemanha: Louis Nebert, 1879, 89 p.
} 
O método por subscritos carrolliano é dotado da plasticidade comum aos trabalhos do período: a mesma notação por subscritos usada para a silogística, onde as letras representam termos, também é usada, sob uma interpretação alternativa, para a lógica proposicional, onde cada letra representa uma proposição atômica.

A aplicação silogística consiste em um método de resolução para silogismos. A teoria silogística carrolliana é uma extensão conservativa da silogística aristotélica (LINDEMANN, 2017) ${ }^{17}$, cuja extensão é fruto da introdução de termos negativos. O uso de termos negativos exige a inclusão da noção de Universo do Discurso, que define a interpretação semântica dos termos negativos, limitando sua extensão ao complemento da extensão do termo positivo em relação ao Universo do Discurso. A relação entre um termo positivo e sua contraparte negativa é dita codivisional.

Segue um exemplo da aplicação do método por subscritos: Considere um silogismo cuja primeira premissa é "Todos os dicionários são úteis", a segunda premissa é "Todos os livros úteis são valiosos" e a conclusão proposta é "Todos os dicionários são valiosos" (CARROLL, 1986, p. 208, tradução nossa) $)^{18}$.

O primeiro passo da aplicação do método consiste em definir o Universo do Discurso do argumento, tomado como sendo "livros" (1986, p. $208)^{19}$. Definir o Universo do Discurso limita a interpretação semântica dos termos de tal modo que, em nosso exemplo, o termo "valiosos" deve ser compreendido como referindo-se apenas a livros valiosos.

O segundo passo consiste na tradução dos termos em língua natural, chamada de forma concreta, para sua respectiva notação por subscritos, chamada de forma abstrata (CARROLL, 1986, p. 109) ${ }^{20}$.

A tabela abaixo associa o possível local de ocorrência dos termos em um silogismo, a forma concreta que ocorre no exemplo e a forma abstrata para a qual devem ser traduzidos. A dupla de termos codivisionais relativa ao termo médio é chamada de Eliminands, dado que o termo médio é eliminado da conclusão, enquanto as outras duas duplas de termos codivisionais são chamados de Retinends, pois um termo de cada um desses pares de termos codivisionais é "retido" na conclusão.

Dado o exemplo proposto, tal como mostra a tabela, devemos assumir que a forma abstrata " $m$ " representa o termo "úteis", " $x$ " representa "dicionários" e " $y$ " representa "valiosos", de tal modo que suas respectivas classes codivisionais, embora não ocorram no silogismo, sejam " $m$ "', que representa o termo negativo "inúteis", " $x^{\prime \prime}$, que representa "não dicionários", e "y'", que representa "não valiosos".

Tabela 1 - A forma abstrata dos termos

\begin{tabular}{|c|c|c|}
\hline Termos no silogismo & $\begin{array}{c}\text { Forma } \\
\text { concreta no } \\
\text { exemplo }\end{array}$ & $\begin{array}{c}\text { Forma } \\
\text { abstrata }\end{array}$ \\
\hline Termo médio positivo (Eliminand) & úteis & $\mathrm{m}$ \\
\hline Termo médio negativo (Eliminand) & inúteis & $\mathrm{m}^{\prime}$ \\
\hline $\begin{array}{l}\text { Termo positivo acompanhando o termo } \\
\text { médio na primeira premissa (Retinend) }\end{array}$ & dicionários & $\mathrm{x}$ \\
\hline
\end{tabular}

${ }^{17}$ LINDEMANN, J. L. A Lógica de Lewis Carroll. Dissertação (Mestrado em Filosofia) - Programa de Pós-graduação em Filosofia, Universidade Federal de Santa Maria, 2017, p. 110-111.

${ }^{18}$ CARROLL, L. Symbolic Logic: Lewis Carroll's. 6 ed. Rev., ampl. e atual. New York: Clarkson Potter, 1986, $514 \mathrm{p}$.

${ }^{19}$ Ibid.

${ }^{20}$ lbid. 


\begin{tabular}{|c|c|c|}
\hline \hline $\begin{array}{c}\text { Termo negativo acompanhanhdo o termo } \\
\text { médio na primeira premissa (Retinend) }\end{array}$ & não dicionários & ' \\
\hline $\begin{array}{c}\text { Termo positivo acompanhanhdo o termo } \\
\text { médio na segunda premissa (Retinend) }\end{array}$ & valiosos & y \\
\hline $\begin{array}{c}\text { Termo negativo acompanhanhdo o termo } \\
\text { médio na segunda premissa (Retinend) }\end{array}$ & não valiosos & $y^{\prime}$ \\
\hline
\end{tabular}

Fonte: Elaborada pelo autor.

O terceiro passo consiste em representar as três proposições do silogismo sob a notação do método por subscritos, compondo uma Formula, onde o sinal " $\dagger$ " representa a conjunção e é usado entre as premissas, enquanto "ף" deve ser compreendido como "se for verdade, então prova que", sendo usado entre as premissas e a conclusão.

A notação por subscritos também utiliza sinais que representam o pressuposto existencial da proposição representada, usando " " para existência e "0" para vacuidade. Assim, " $x_{1}$ " representa a proposição "Algum $x$ existe", isto é, a extensão do termo x não é vazia, denominando tal proposição de Entidade, enquanto " $\mathrm{x}_{0}$ " significa "Nenhum $\mathrm{x}$ existe", isto é, a extensão do termo x é vazia, denominando tal proposição de Nulidade.

Dado os sinais para os pressupostos existenciais, segue-se que "Algum $x$ é $y$ " é representada por " $\mathrm{xy}_{1}$ ". Note que " $\mathrm{xy}_{1}$ " e " $\mathrm{yx}_{1}$ " são equivalentes, assim como "Algum $x$ é $y$ " é equivalente a "Algum y é $x$ ". "Nenhum $x$ é y" é representada, mutatis mutandis, por " $\mathrm{xy}_{0}$ " ou " $\mathrm{yx}_{0}$ ".

Cabe especificar que, dado o uso de termos negativos, Carroll dispensa o uso de proposições Particulares Negativas, onde "Algum x não é m" deve ser compreendida como "Algum $x$ é $\mathrm{m}^{\prime \prime}$ ", supondo o mesmo pressuposto existencial das Particulares Afirmativas, isto é, " $x m^{\prime}{ }_{1}$ " ou " $m^{\prime} x_{1}$ ".

Diferente dos lógicos contemporâneos, Carroll não aceita que proposições Universais Afirmativas possam ser satisfeitas por vacuidade (LINDEMANN, 2017, p. 63-72) ${ }^{21}$, considerando proposições Universais Afirmativas como proposições duplas, cujo conteúdo pode ser expresso por outras duas proposições sem qualquer perda ou acréscimo de informação, por exemplo, "Todo $x$ é $m$ " pode ser expresso pelas proposições "Algum x é $m$ " e "Nenhum $x$ é $\mathrm{m}$ " ".

Deste modo, "Todo $x$ é $m$ " é equivalente a " $x m_{1} \dagger \times m^{\prime}{ }^{\prime}$ ", podendo ser escrita de forma curta como " $\mathrm{x}_{1} \mathrm{~m}^{\prime}{ }_{0}$ ". Carroll utiliza apenas " $\mathrm{x}_{1}$ " ao invés de " $\mathrm{xm}_{1}$ " graças ao limite semântico imposto pelo Universo do Discurso, dado que " $\mathrm{xm}_{1} \dagger \mathrm{xm}_{0}{ }_{0}$ " implica " $\mathrm{x}_{1}$ " e " $\mathrm{m}_{0}{ }^{\prime}$ " (CARROLL, 1986, p. 121) ${ }^{22}$. Note que $\mathrm{O}$ termo " $m$ " não ocorre na representação por subscritos de "Todo $x$ é $m$ ", onde a informação da relação que " $m$ " mantém com " $x$ ", tal como expressa na proposição, é contemplada pela ocorrência da nulidade de seu termo codivisional na notação por subscritos.

Cada proposição representada sob a notação por subscritos será considerada como Entidade ou Nulidade de acordo com o sinal existencial do segundo termo, se for " 1 ", então a proposição será uma Entidade, se for " 0 ", será uma Nulidade. Por exemplo, a representação por subscritos da proposição "Todo $x$ é $m$ ", isto é, " $\mathrm{x}_{1} \mathrm{~m}_{0}$ ", é uma Nulidade.

Abaixo, as três proposições que compõem o silogismo do exemplo, agora com os termos em forma abstrata, ao lado de sua respectiva Formula, isto é, sua expressão sob a notação por subscritos. Repare que as três

${ }^{21}$ LINDEMANN, J. L. A Lógica de Lewis Carroll. Dissertação (Mestrado em Filosofia) - Programa de Pós-graduação em Filosofia, Universidade Federal de Santa Maria, 2017, p. 63-72.

${ }^{22}$ CARROLL, L. Symbolic Logic: Lewis Carroll's. 6 ed. Rev., ampl. e atual. New York: Clarkson Potter, $1986,514 \mathrm{p}$. 
proposições do exemplo são Universais Afirmativas, consideradas proposições duplas por Carroll, não sendo representadas diretamente na Formula, mas através da representação da dupla de proposições que expressa o seu conteúdo. Por exemplo, a primeira premissa, a saber, "Todo x é m", cujo conteúdo é expresso pela dupla de proposições "Algum x é m" e "Nenhum x é $\mathrm{m}^{\prime \prime}$, é representada por " $\mathrm{x}_{1} \mathrm{~m}_{0}$ " na Formula.

Todo $\mathrm{x}$ é $\mathrm{m}$.

Todo m é $\mathrm{y}_{\text {. }} \quad=\quad \mathrm{x}_{1} \mathrm{~m}^{\prime}{ }_{0} \uparrow \mathrm{m}_{1} \mathrm{y}^{\prime}{ }_{0}$ ๆ $\mathrm{x}_{1} \mathrm{y}^{\prime}{ }_{0}$

Todo $x$ é y.

O quarto passo da aplicação do método por subscritos consiste na identificação de certas características da Formula analisada, enquadrando-a ou não como pertencente a uma das três Figuras, apresentadas abaixo.

Dada uma Formula qualquer, se a letra que representa a forma abstrata de qualquer par de termos codivisionais ocorre duas vezes como positiva ou duas vezes como negativa, ela é chamada de Like, mas se ocorre uma vez como positiva e outra como negativa, chama-se Unlike. No exemplo de Formula acima, " $x$ " e " $y$ " são Like, enquanto " $m$ " é Unlike, dado que ocorre como " $\mathrm{m}$ " na primeira premissa e como " $\mathrm{m}$ " na segunda.

Todos os silogismos válidos dividem-se em três tipos de Formulas, chamadas por Carroll de Figuras, especificando regras para que se identifique a qual Figura cada Formula pertence (CARROLL, 1986, p. 123-126) ${ }^{23}$.

Identifica-se Formulas que pertencem a Figura 1, do tipo " $\mathrm{xm}_{0}+\mathrm{ym}_{{ }_{1}}$ q xyo", constatando que duas Nulidades, com Eliminands Unlike, geram uma Nulidade, onde todos os Retinends são Like. Caso um Retinend tenha sua existência afirmada em uma premissa, também será afirmada na conclusão.

Identifica-se Formulas que pertencem a Figura 2, do tipo " $\mathrm{xm}_{0} \dagger \mathrm{ym}_{1}$ q $x^{\prime} y_{1}{ }^{\prime \prime}$, constatando que uma Nulidade e uma Entidade, com Eliminands Like, geram uma Entidade, onde o Retinend da Nulidade é Unlike.

Identifica-se Formulas que pertencem a Figura 3, do tipo " $x m_{0} \dagger m_{1} y_{0}$ ๆ $\mathrm{x}^{\prime} \mathrm{y}_{1}{ }_{1}$ ", constatando que duas Nulidades, com Eliminands Like, geram uma Entidade, onde os dois Retinends são Unlike.

A Formula do exemplo analisado, a saber, " $\mathrm{x}_{1} \mathrm{~m}^{\prime}{ }_{0}+\mathrm{m}_{1} \mathrm{y}_{0}{ }_{0}$ ๆ $\mathrm{x}_{1} \mathrm{y}_{0}{ }_{0}$ ", é identificada como uma Formula da Figura 1, dado que todos os Retinends são Like, duas premissas são Nulidades com Eliminands Unlike e a conclusão também é uma Nulidade. Repare que o Retinend " $x$ " tem sua existência afirmada na primeira premissa, tal como na conclusão.

Ao reconheceremos a Formula do exemplo como pertencente à Figura 1, diagnostica-se a validade do silogismo analisado. Assim, o método por subscritos aplicado à silogística consiste na tradução do silogismo para uma Formula que pode ser identificada como pertencente a uma das três Figuras, caso a Formula pertença a uma Figura, constata-se que o silogismo é válido, caso não pertença a nenhuma Figura, constata-se que o silogismo é falacioso.

Diferente da leitura silogística, Carroll não nos deixou uma exposição sistemática da leitura proposicional de seu método por subscritos, apenas escassos exemplos de sua aplicação, que vieram a público com a publicação póstuma de "Symbolic Logic: Part II" (CARROLL, 1986) ${ }^{24}$.

Deve-se compreender proposição, tal como definido por Carroll (1986, p. 67) ${ }^{25}$, como qualquer frase que transmite alguma informação, seja ela qual for. A noção de proposição só recebeu uma definição mais clara em épocas

${ }^{23}$ CARROLL, L. Symbolic Logic: Lewis Carroll's. 6 ed. Rev., ampl. e atual. New York: Clarkson Potter, $1986,514 \mathrm{p}$.

${ }^{24}$ CARROLL, L. Symbolic Logic: Lewis Carroll's. 6 ed. Rev., ampl. e atual. New York: Clarkson Potter, $1986,514 \mathrm{p}$.

${ }^{25}$ lbid. 
posteriores, através dos trabalhos de Frege, Russell e Moore (McGRATH, $2012)^{26}$. A definição carrolliana será interpretada como congruente com a que estava sendo usada pelos seus pares a partir das inovações de Boole (1854) ${ }^{27}$, considerando que a toda informação pode-se atribuir "verdadeiro" ou "falso".

Quando aplicado a lógica proposicional, cada letra passa a representar uma proposição atômica e compreende-se a apóstrofe como negação, onde uma letra com apóstrofe representa a proposição negada. As letras usadas para representar proposições não se limitam a " $x$ ", " $y$ " e " $m$ ", mas estendemse a todo o alfabeto.

O sinal " $\dagger$ " continua sendo usado como conjunção, mas a simples justaposição entre duas letras também deve ser compreendida como uma conjunção entre elas, e "q" passa a ser usado como implicação material. O sinal "§" é introduzido como disjunção inclusiva, enquanto " $\equiv$ " é introduzido como sinal de equivalência (CARROLL, 1986, p. 256) ${ }^{28}$. Tal como no cálculo proposicional clássico, parênteses são usados como recurso sintático.

Diferente da aplicação silogística, os números " 1 " e "0" não ocorrem em todas as Formulas sob leitura proposicional, mas, quando ocorrem, passam a exercer uma função metalógica (semântica), onde " ${ }_{1}$ " pode ser compreendido como a atribuição de verdade para a proposição na qual está associado, enquanto "o" atribui a falsidade.

Utilizar símbolos com funções semânticas não é uma idiossincrasia de Carroll. Alguns sistemas lógicos utilizam " $T$ " como uma constante para 0 verdadeiro e " $\perp$ " como uma constante para o falso ou absurdo (GABBAY; GUENTHNER, 2002, p. 12) $)^{29}$. Em tais sistemas, " $\neg P$ ", isto é, "não $P$ ", pode ser definido como " $\mathrm{P} \rightarrow \perp$ ".

Segue um exemplo de Formula do método por subscritos em seu uso proposicional: " $\left(a b_{0}\right) \equiv\left(a_{1}{ }_{1} \S b_{1}{ }_{1}\right)$ ". Tal Formula pode ser expressa, sob a notação do cálculo proposicional clássico, com o acréscimo dos símbolos " $T$ " como constante para verdadeiro e " $\perp$ " como constante para o falso, da seguinte forma: " $((A \wedge B) \rightarrow \perp) \leftrightarrow((\neg A \rightarrow T) \vee(\neg B \rightarrow T))$ ". A mesma Formula, traduzida para a língua natural, expressa que a conjunção de $A$ e $B$ é falsa se, e somente se, não-A é verdadeiro ou não-B é verdadeiro.

\section{Pseudomenos}

Pseudomenos é um paradoxo clássico, também conhecido como "paradoxo do mentiroso", cuja formulação embrionária é tradicionalmente atribuída a Epimênides, que viveu em meados de 600 a.C. Lewis Carroll apresenta o paradoxo da seguinte forma:

Se um homem diz "Eu estou dizendo uma mentira", e fala a verdade, ele está falando uma mentira, portanto sua fala é falsa: mas se o que ele fala é falso, ele não está mentindo, portando sua fala é verdadeira. $\left(1986\right.$, p. 425, tradução nossa) ${ }^{30}$

\footnotetext{
${ }^{26}$ McGRATH, M. "Propositions". In: ZALTA, E. Z. et al (Org.). Stanford Encyclopedia of Philosophy. Stanford, California. 20 Jun. 2012. http://plato.stanford.edu/entries/propositions/ (último acesso em 28/08/17).

${ }^{27} \mathrm{BOOLE}, \mathrm{G}$. An Investigation of the Laws of Thought: on which are founded: The Mathematical Theories of Logic and Probabilities. Cambridge: McMillan and Co., 1854, 425 p.

${ }^{28}$ CARROLL, L. Symbolic Logic: Lewis Carroll's. 6 ed. Rev., ampl. e atual. New York: Clarkson Potter, 1986, 514 p.

${ }^{29}$ GABBAY, D.M.; GUENTHNER, F. (Eds.). Handbook of Philosophical Logic: Volume 6. 2 ed. New York: Springer, 2002, p. 12.

${ }^{30}$ CARROLL, L. Symbolic Logic: Lewis Carroll's. 6 ed. Rev., ampl. e atual. New York: Clarkson Potter, 1986, $514 \mathrm{p}$.
} 
O tratamento lógico dado ao paradoxo utiliza a leitura proposicional do método por subscritos (CARROLL, 1986, p. 434-436) ${ }^{31}$, com a consideração prévia de que seja razoável supor que a proposição “Eu estou dizendo uma mentira" refere-se a si mesma como seu próprio assunto.

O Universo do Discurso é definido como "Cosmophases" (CARROLL, 1986, p. 435) ${ }^{32}$. Embora o que Carroll compreende como "Universo do Discurso" de argumentos proposicionais não tenha sido especificado pelo autor, podemos supor que se refira ao evento ou ao estado de coisas segundo o qual as proposições de um argumento reivindicam verdade ou falsidade, onde a exótica definição utilizada deve ser compreendida como um limite semântico fixado na "fase cósmica", isto é, "algum momento particular" (CARROLL, 1986, p. 481) ${ }^{33}$ no qual um certo homem enuncia a proposição "Eu estou dizendo uma mentira".

A forma abstrata "a" é usada para representar a proposição "O homem fala a verdade", enquanto a forma abstrata " $b$ " é usada para representar a proposição "A proposição, estabelecida pelo homem, pode ser seu próprio assunto".

Dada as formas abstratas para a proposição paradoxal e para a proposição que explicita sua suposição de autorreferência, segue-se "a ी a'b", isto é, "Se o homem fala a verdade, então o homem não fala a verdade e a proposição, estabelecida pelo homem, pode ser seu próprio assunto", e "a'b q a", isto é, "Se o homem não fala a verdade e a proposição que estabeleceu é seu próprio assunto, então o homem fala a verdade".

Segundo Carroll ${ }^{34}$ (1986, p. 435), "(a I $\left.a^{\prime} b\right)$ † (a'b ी a)" provam “b" isto é, provam a falsidade da proposição "A proposição, estabelecida pelo homem, pode ser seu próprio assunto", estabelecendo a falsidade da hipótese de autorreferência da proposição paradoxal.

Carroll não demonstra como “( $a$ ๆ $a$ 'b) † ( $a$ 'b ๆ $a)$ " provam “ $b_{0}$ ”, mas a validade de tal inferência pode ser facilmente demonstrada pelo método de dedução natural aplicado ao cálculo proposicional clássico, como se segue, onde " $A$ " representa a proposição "Eu estou dizendo uma mentira" e " $B$ " representa a proposição "A proposição, estabelecida pelo homem, pode ser seu próprio assunto".

A dedução natural abaixo utiliza uma notação tradicional e regras clássicas de inferência, onde um hífen ("“-") indica que as fórmulas que ocorrem na linha supõem uma hipótese.

1. $(A \rightarrow(\neg A \wedge B)) \wedge((\neg A \wedge B) \rightarrow A)$ : Premissa.

2. $A \rightarrow(\neg A \wedge B)$ : de 1 , por Eliminação da Conjunção.

3. $(\neg A \wedge B) \rightarrow A$ : de 1 , por Eliminação da Conjunção.

4. - A: Hipótese para Redução ao Absurdo.

5. $\quad-\neg A \wedge B$ : de 2 e 4 , por Modus Ponens.

6. $\quad-\neg A$ : de 5 , por Eliminação da Conjunção.

7. $-A \wedge \neg A$ : de 4 e 6 , por Introdução da Conjunção.

8. $\neg \mathrm{A}$ : de 4 a 7 , por Redução ao Absurdo.

9. - B: Hipótese para Redução ao Absurdo.

10. - $\neg A \wedge B$ : de 8 e 9 , por Introdução da Conjunção.

11. - A: de 3 e 10, por Modus Ponens.

12. - $A \wedge \neg A$ : de 8 e 11 , por Introdução da Conjunção.

13. $\neg$ B: de 9 a 12, por Redução ao Absurdo.

\footnotetext{
${ }^{31}$ Ibid.

${ }^{32}$ Ibid.

${ }^{33}$ Ibid.

${ }^{34}$ CARROLL, L. Symbolic Logic: Lewis Carroll's. 6 ed. Rev., ampl. e atual. New York: Clarkson Potter, $1986,514 \mathrm{p}$.
} 
A dedução natural acima demonstra que “ $\neg B$ " se segue de “ $(A \rightarrow(\neg A \wedge$ $B)) \wedge((\neg A \wedge B) \rightarrow A)$ ", isto é, utilizando a notação por subscritos carrolliana,

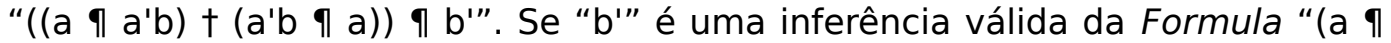
$\left.a^{\prime} b\right)+(a ' b$ ๆ $a)$ ", então tal Formula prova "bo", como suposto por Carroll, provando a falsidade da proposição "A proposição, estabelecida pelo homem, pode ser seu próprio assunto".

Se a proposição "Eu estou dizendo uma mentira" não pode estar referindo-se a si mesma como seu próprio assunto, devemos supor que referese a outra proposição qualquer, que nos é oculta, mas que foi enunciada pelo mesmo homem no mesmo contexto conversacional, ou "Cosmophases".

A proposição oculta que é o assunto do enunciado "Eu estou dizendo uma mentira" pode ser falsa, no caso do enunciado ser verdadeiro, ou verdadeira, no caso do enunciado ser falso, mas em ambos os casos já não há qualquer implicação paradoxal.

Uma solução distinta, mas com resultado semelhante à proposta por Carroll, deriva-se dos trabalhos de Alfred Tarski (1901-1983). Tarski propõe uma hierarquia entre linguagens e metalinguagens segundo a qual nenhuma linguagem pode conter proposições que prediquem a verdade ou a falsidade de proposições da própria linguagem, isto é, em sua própria terminologia, nenhuma linguagem pode ser semanticamente fechada (BEALL; GLANZBERG; RIPLEY, 2016) ${ }^{35}$.

Por exemplo: utilizando uma certa linguagem $L$ não se pode predicar a verdade ou a falsidade de proposições da própria linguagem $L$, apenas utilizando uma metalinguagem $L^{\prime}$ é que se pode predicar a verdade ou falsidade de proposições da linguagem $L$, assim como não se pode predicar o valor de verdade de proposições de L' com a própria linguagem L', apenas com uma metalinguagem L".

Dada a hierarquia de Tarski, segue-se que a proposição "Eu estou dizendo uma mentira" não pode ser autorreferente, pois nenhuma linguagem pode ser semanticamente fechada.

A solução do paradoxo oriunda da teoria de Tarski deriva-se das implicações de sua hierarquia entre linguagens e metalinguagens, enquanto a solução carrolliana deriva-se da consequência lógica da relação entre a proposição paradoxal e a hipótese de que ela seja autorreferente, mas ambas dissolvem o paradoxo concluindo que a proposição "Eu estou dizendo uma mentira" não pode, necessariamente, referir-se a si mesma como seu próprio assunto.

Embora cheguem à mesma solução, a abordagem carrolliana é mais simples na medida em que se segue de uma consequência lógica da relação entre a proposição paradoxal e sua hipótese de autorreferência, sem comprometer-se com a defesa de uma teoria semântica da verdade, tal como a abordagem de Tarski, cuja teoria já foi atacada por Smullyan (1957, p. 55$67)^{36}$, que apresenta uma linguagem semanticamente fechada como contraexemplo à sua hierarquia entre linguagens e metalinguagens.

\section{Antistrephon}

Antistrephon, também conhecido como "paradoxo do advogado" ou "paradoxo de Protágoras", é um paradoxo clássico, cujas raízes remontam à Grécia Antiga. O paradoxo é apresentado por Lewis Carroll da seguinte forma:

${ }^{35}$ BEALL, J; GLANZBERG. M; RIPLEY, D. "Liar Paradox". In: ZALTA, E. Z. et al (Org.). Stanford Encyclopedia of Philosophy. Stanford, California. 12 Dez. 2016.

https://plato.stanford.edu/entries/liar-paradox/\#TarsHierLang (último acesso em 03/09/17)

${ }^{36}$ SMULLYAN, R. M. "Languages in Which Self Reference is Possible". In: The Journal of Symbolic Logic. Cambridge, v. 22, n. 1, 1957, p. 55-67. 
[Antistrephon,] ou seja, A Réplica. Este é um conto sobre tribunais. Protágoras havia combinado de treinar Euathius para a profissão de advogado, sob a condição de que metade do pagamento deveria ser realizada imediatamente e de que a outra metade seria paga, ou não precisaria ser paga, de acordo com Euathius ganhar, ou perder, o seu primeiro caso na corte. Depois de um tempo, Protágoras, começando a ficar impaciente, entra com uma ação contra o seu pupilo, visando recuperar a segunda parte do seu pagamento. Mas Euathis decide pleitar o seu [primeiro e] próprio caso. "Agora, se eu ganhar esta ação", diz Protágoras, "você deverá pagar o dinheiro pela decisão da corte, mas, se eu perder, você deverá pagar devido ao nosso acordo. Ou seja, em qualquer caso, você deverá me pagar." "Pelo contrário," replicou Euathius, "se você ganhar esta ação, eu serei liberado do pagamento pelo nosso acordo, mas, se você perder, eu serei liberado pela decisão da corte. Ou seja, em qualquer caso, eu não precisarei pagar o dinheiro". (CARROLL, 1986, p. 426, tradução nossa) ${ }^{37}$

Carroll oferece apenas um tratamento lógico informal para o paradoxo, assumindo que o acordo e a decisão da corte causam um excesso de regras, de tal modo que a solução consiste em compreender o que resulta da colisão de tais regras (CARROLL, 1986, p. 438) (38. $^{38}$

Deriva-se duas hipóteses do excesso de regras:

(1) Podemos supor que o acordo é supremo. Neste caso, se Protágoras ganhar a ação, ele não ganhará o dinheiro, mas se ele perder a ação, então ele deve receber o dinheiro.

(2) Podemos supor que a decisão da corte é suprema. Neste caso, se Protágoras ganhar a ação, ele ganhará o dinheiro, mas se perder a ação, então não ganhará o dinheiro.

Os dados não nos permitem escolher qual é a melhor hipótese, mas Carroll acredita que a decisão da corte será obviamente contra Protágoras, pois os requisitos do acordo ainda não foram cumpridos. Quando a decisão for pronunciada, se (1), então Euathius deverá pagar, se (2), então ele estará livre do pagamento.

Segue uma possível formalização da solução sob a notação do cálculo proposicional clássico, onde " $A$ " representa a proposição "O acordo é supremo", "B" representa "Protágoras ganha a ação" e "C" representa "Protágoras ganha o dinheiro".

$$
((\mathrm{A} \wedge \mathrm{B}) \rightarrow \neg \mathrm{C}) \wedge((\mathrm{A} \wedge \neg \mathrm{B}) \rightarrow \mathrm{C}) \wedge((\neg \mathrm{A} \wedge \mathrm{B}) \rightarrow \mathrm{C}) \wedge((\neg \mathrm{A} \wedge \neg \mathrm{B}) \rightarrow \neg \mathrm{C})
$$

Embora a solução carrolliana para o paradoxo do advogado não tenha sido apresentada de maneira formal pelo autor, aos moldes da fórmula acima, expressa sob a notação do cálculo proposicional, é possível propor a seguinte Formula sob a leitura proposicional do método por subscritos:

$$
\left.\left(a b \text { ๆ } c^{\prime}\right)+\left(a b^{\prime} \text { ๆ } c\right) \text { † }\left(a^{\prime} b \text { ๆ } c\right) \text { † ( } a^{\prime} b^{\prime} \text { ๆ } c^{\prime}\right)
$$

Leibniz (1646-1716) também apresentou uma solução para o paradoxo, mas, diferente de Carroll, não considera que haja um excesso de regras e sequer considera que o caso seja perplexo. A solução de Leibniz, na obra "Dissertatio Inauguralis de Casibus Perplexis in Juri" (1666 apud ARTOSI; PIERI; SARTOR, 2013, p. xx-xxii) ${ }^{39}$, é a seguinte: A decisão da corte deve ser contra Protágoras, dado que as condições do acordo ainda não foram cumpridas, livrando Euathius do pagamento. Assim que a sentença for proferida,

${ }^{37}$ CARROLL, L. Symbolic Logic: Lewis Carroll's. 6 ed. Rev., ampl. e atual. New York: Clarkson Potter, $1986,514 \mathrm{p}$.

${ }^{38}$ Ibid.

${ }^{39}$ ARTOSI, A; PIERI, B; SARTOR, G. (Eds.). Leibniz: Logico-Philosophical Puzzles in the Law:

Philosophical Questions and Perplexing Cases in the Law. New York: Springer, 2013, p. xx-xxii. 
Protágoras deve entrar com uma nova ação judicial, que será aceita, pois não é idêntica à anterior, dado que há um fato novo e relevante, a saber, a própria decisão anterior. Protágoras ganhará a segunda ação, pois agora as condições do acordo já foram cumpridas, e Euathius deverá pagar seu mestre, respeitando a equidade natural e a rigorosidade da lei.

Se comparada à solução carrolliana, percebe-se que Leibniz julga que a decisão da corte será suprema e que Protágoras perderá o caso, hipótese contemplada pelo último conjuntivo da Formula carrolliana, a saber, "a'b' १ $C^{\prime \prime \prime}$. Com tal pressuposto, Leibniz soluciona o impasse prescrevendo a próxima ação de Protágoras, enquanto a solução de Carroll limita-se a elencar as possibilidades lógicas de resolução do caso.

\section{5. "On the way to the barber-shop"}

Controvérsias sobre a natureza da implicação remontam à Grécia Antiga, especificamente a um famoso debate entre Filo de Megara ( $f$. 300 a.C.), defendendo que a implicação é verdadeira em todos os casos em que o antecedente é falso ou o consequente verdadeiro, mesmo que casualmente, e Diodoro Cronos (ca. 405-304 a.C.), defendendo que a relação de implicação entre antecedente e consequente deve possuir um vínculo modal de necessidade (OHRSTROM; HASLE, 1995, p. 19-20) . $^{40}$

Dois milênios depois da controvérsia original, foi “No caminho para a barbearia" (CARROLL, 1986, p. 428-431, tradução nossa) ${ }^{41}$, um quebracabeças carrolliano, que pautou os debates sobre a natureza da implicação que deram luz à interpretação verofuncional de implicação adotada pelos lógicos contemporâneos. Segundo Bartley III (1986, p. 444-445) ${ }^{42}$, essa é uma das mais curiosas anomalias lógicas tratadas por Carroll, que nos deixou oito versões diferentes do problema, tendo gerado uma vasta literatura que o discute, sendo "descrito repetidamente por Boole, Jevons, Venn, John Neville Keynes e outros como 'o problema central da lógica simbólica'" (BARTLEY III, 1986, p. 445, tradução nossa) ${ }^{43}$.

Carroll apresentou o problema através de diferentes histórias ornamentais, mas, segundo o autor, ele surgiu de uma controvérsia entre dois estudantes de lógica no ano de 1893 (CARROLL, 1986, p. 442) ${ }^{44}$, consistindo, em síntese, na seguinte forma:

Dadas três proposições, representadas por A, B e C.

Nós temos:

(1) Se C é verdadeira, segue-se que se A é verdadeira, então B não é verdadeira. nossa $)^{45}$

(2) Se A é verdadeira, então $B$ é verdadeira.

Será que C pode ser verdadeira? (CARROLL, 1986, p. 442, tradução

Embora não seja um paradoxo lógico genuíno, o ponto que estava em disputa na época era "se duas condicionais, na forma 'se $A$, então $B$ ' e 'se $A$, então não-B' são compatíveis" (CARROLL, 1986, p. 444, tradução nossa) ${ }^{46}$,

${ }^{40}$ OHRSTROM, P.; HASLE, P. F. V. Temporal Logic: From Ancient Ideas to Artificial Intelligence. Dordrecht, Holanda: Kluwer Academic Publishers, 1995, p. 19-20.

${ }^{41}$ CARROLL, L. Symbolic Logic: Lewis Carroll's. 6 ed. Rev., ampl. e atual. New York: Clarkson Potter, 1986, 514 p.

${ }^{42}$ BARTLEY III. W. W. "Annotations". In: CARROLL, L. Symbolic Logic: Lewis Carroll's. 6. ed. Rev.,

ampl. e atual. New York: Clarkson Potter, 1986, 514 p.

${ }^{43}$ Ibid.

${ }^{44}$ CARROLL, L. Symbolic Logic: Lewis Carroll's. 6 ed. Rev., ampl. e atual. New York: Clarkson Potter, 1986, $514 \mathrm{p}$.

${ }^{45}$ Ibid.

${ }^{46}$ Ibid. 
fazendo com que seu aspecto paradoxal provenha da possível verdade de " $C$ " gerar a aparente contradição entre as implicações "Se A, então não-B" em (1) e "Se A, então B" em (2).

A fim de evitar uma leitura anacrônica, deve-se realizar uma interpretação cuidadosa da questão. O problema expressa em (2) que, em condições normais, "A" implica " $B$ ", mas expressa em (1) que, caso "C" seja verdadeira, então " $A$ " implica "não-B". Assim, o cerne consiste em saber se há uma possível proposição " $C$ " cuja verdade faça com que " $A$ ", que implica " $B$ ", também implique "não-B".

Muitos lógicos do período da álgebra da lógica (BARTLEY III, 1986, p. 449) ${ }^{47}$, incluindo John Cook Wilson (1849-1915), argumentaram que se " $A$ " implica " $B$ ", então é um absurdo que " $A$ " também possa implicar "não-B", defendendo ser impossível que haja um caso no qual " $C$ " seja verdadeira $e$ todas as condicionais satisfeitas (CARROLL, 1986, p. 450-462) ${ }^{48}$.

Carroll, por sua vez, argumentou em prol da possível verdade de " $C$ ", apresentando um exemplo de caso que visa demonstrar tal possibilidade lógica, contrariando a posição de John Cook Wilson. O exemplo de caso apresentado por Carroll (1986, p. 444) ${ }^{49}$ consiste em uma interpretação semântica das proposições "A", "B" e " $C$ " na qual cada proposição expressa certas propriedades associadas a uma figura geométrica composta pelos pontos "K", "L", "M" e "N", pelas linhas "KL", "LM" e "MN", e pelos ângulos " $\angle K L M "$ e " $\angle L M N "$, assumindo que os dois ângulos sejam iguais do mesmo lado da linha "LM".

Figura 1 - Uma figura aberta

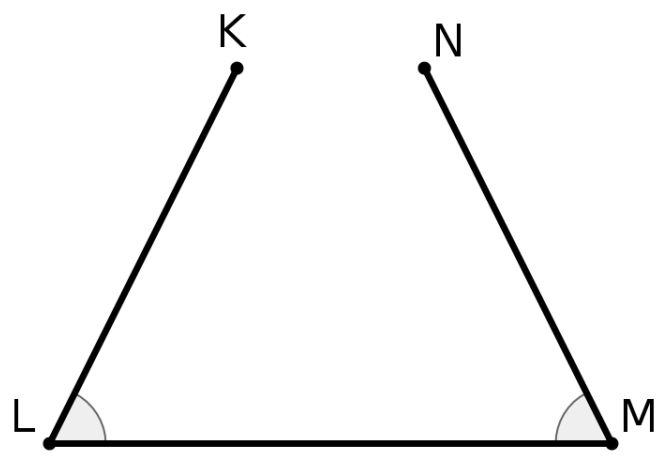

Fonte: Elaborada pelo autor, baseada em Carroll (1986, p. 444) ${ }^{50}$.

Em referência a tal figura geométrica, Carroll (1986, p. 444) ${ }^{51}$ toma "A" como representando a proposição atômica "Os pontos $\mathrm{K}$ e $\mathrm{N}$ coincidem de modo que as três linhas formam um triângulo", "B" como representando "Os ângulos $\angle K L M$ e $\angle L M N$ são iguais" e "C" como representando "As linhas KL e MN não são iguais".

Analisando a figura em questão e os dados sobre ela fornecidos em sua apresentação textual, constata-se que a proposição " $C$ " pode ser verdadeira ou falsa, pois não dispomos de informações que sejam suficientes para conhecer o seu valor de verdade, mas reconhecemos que " $A$ " é falsa, dado que trata-se de uma figura aberta na qual os pontos $\mathrm{K}$ e $\mathrm{N}$ visivelmente não

\footnotetext{
${ }^{47}$ BARTLEY III. W. W. "Annotations”. In: CARROLL, L. Symbolic Logic: Lewis Carroll's. 6. ed. Rev., ampl. e atual. New York: Clarkson Potter, 1986, 514 p.

${ }^{48}$ CARROLL, L. Symbolic Logic: Lewis Carroll's. 6 ed. Rev., ampl. e atual. New York: Clarkson Potter, $1986,514 \mathrm{p}$.

${ }^{49}$ Ibid.

${ }^{50}$ Ibid.

${ }^{51}$ CARROLL, L. Symbolic Logic: Lewis Carroll's. 6 ed. Rev., ampl. e atual. New York: Clarkson Potter, $1986,514 \mathrm{p}$. 
coincidem, e "B" é verdadeira, o que é especificado pela apresentação textual da figura em questão.

Carroll não usou o seu método por subscritos em suas publicações sobre "No caminho para a barbearia" (a leitura proposicional do método só veio a público postumamente), mas seu uso nos ajuda a compreender a abordagem lógica do autor.

Diferente da formalização do problema sob a notação do Cálculo Proposicional Clássico, a saber, " $(C \rightarrow(A \rightarrow \neg B)) \wedge(A \rightarrow B)$ ", o método por subscritos carrolliano, graças ao uso de símbolos que atuam como constantes para o valor de verdade das proposições, possibilita que o problema seja expresso sob uma Formula em que todas as considerações sobre os valores de verdade da apresentação informal originária do problema tenham um correlato em sua forma lógica, dispensando o uso da negação, tal como se segue, onde cada proposição será representada por sua respectiva letra minúscula:

$\left(c_{1}\right.$ ๆ $\left(a_{1}\right.$ ๆ $\left.\left.b_{0}\right)\right)+\left(a_{1}\right.$ ๆ $\left.b_{1}\right)$

Devido ao caráter hipotético da implicação, a Formula acima especifica o valor de verdade da ocorrência de cada proposição tal como suposto às relações condicionais do problema, independente dos valores de verdade reais de cada proposição oriundos da interpretação semântica proposta por Carroll, segundo a qual o valor de "c" é desconhecido, "b" é verdadeira e "a" é falsa, isto é, "c", "b1" e "a0".

A relação condicional do segundo conjuntivo da Formula acima, a saber, " $\left(a_{1} \cap b_{1}\right)$ ", dita que se "a" for verdadeira, então "b" será verdadeira. Sabemos que o consequente " $b$ " é verdadeiro, tal como especificado pela apresentação textual da figura, e que o antecedente "a" é falso, pois a figura obviamente não é um triângulo, mas o caráter hipotético da condicional exige que examinemos se a hipótese de verdade do antecedente mantém a verdade do consequente. Sua tradução para a forma concreta, dada a interpretação semântica proposta, expressa que se a figura é um triângulo de modo que os pontos $\mathrm{K}$ e $\mathrm{N}$ coincidam, então os ângulos $\angle K L M$ e $\angle L M N$ são iguais.

Figura 2 - Triângulo isósceles

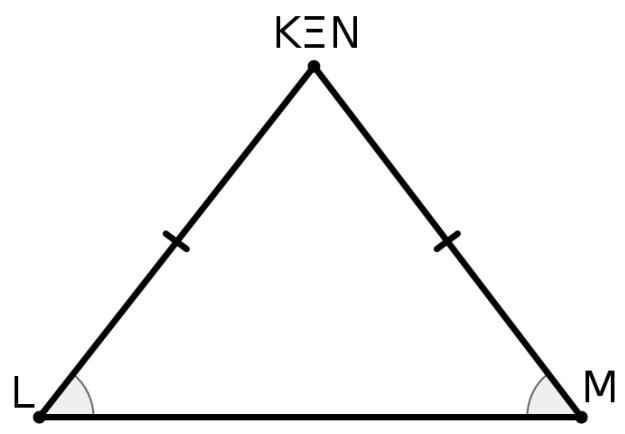

Fonte: Elaborada pelo autor.

Segue-se que, sem qualquer consideração acerca do valor de verdade de " $c$ ", a hipótese da verdade de "a" estabelece a verdade de " $b$ ", dado que o conhecimento prévio da verdade de "b" nos orienta a compreender a figura como um triângulo isósceles, de modo que os pontos $\mathrm{K}$ e $\mathrm{N}$ coincidam e os ângulos $\angle K L M$ e $\angle L M N$ sejam iguais, como na figura acima.

Por outro lado, as relações condicionais expressas pelo primeiro conjuntivo da Formula, a saber, " $\left(c_{1}\right.$ ๆ $\left(a_{1}\right.$ ๆ $\left.\left.b_{0}\right)\right)$ ", ditam que se " $c$ " for 
verdadeira, então se "a" for verdadeira, "b" será falsa. Isto é, assumindo a verdade de " $c$ ", segue-se que se "a" for verdadeira, então " $b$ " será falsa.

Sabemos que não é o caso que "a" seja verdadeira e "b" falsa, mas isto não está em questão, e sim se, supondo a verdade de "c", a hipótese da verdade de "a" implica a falsidade de "b".

Ao supormos a verdade de "a", isto é, que os pontos $\mathrm{K}$ e $\mathrm{N}$ coincidam de modo que a figura seja um triângulo, a proposição " $b$ " só é verdadeira, isto é, os ângulos $\angle K L M$ e $\angle L M N$ só são iguais, caso as linhas $K L$ e $M N$ também sejam iguais, como na figura 2 , justamente o que é negado por "c", cuja verdade foi previamente assumida, a saber, que as linhas $\mathrm{KL}$ e $M N$ não são iguais. Segue-se que se supormos a verdade de "c" e "a", então os ângulos $\angle K L M$ e $\angle L M N$ precisam ser distintos, isto é, "b" precisa ser falsa, tal como exemplificado pela figura abaixo.

Figura 3 - Triângulo com linhas KL e MN tendo tamanhos distintos

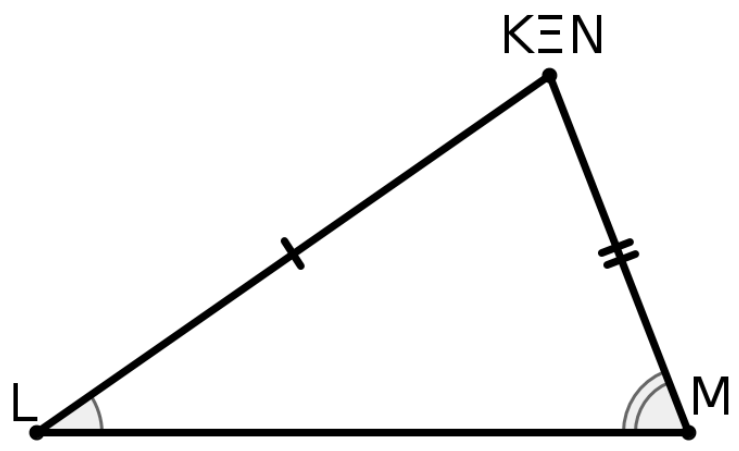

Fonte: Elaborada pelo autor.

A interpretação semântica proposta por Carroll não especifica o valor de verdade de "C", mas dita que " $B$ " é verdadeira e " $A$ " é falsa, lembrando que seu objetivo consiste em demonstrar que "A implica B" é compatível com "A implica não-B". A condicional "Se A, então B" é satisfeita supondo apenas os dados extraídos da figura e de sua apresentação textual, enquanto a condicional "Se A, então não-B" é satisfeita quando acrescentamos a esses dados a hipótese da verdade de " $C$ ". Segue-se que a suposta verdade de "C" faz com que a proposição "A", que implica "B", passe a implicar "não-B".

O exemplo carrolliano, assumindo a falsidade de " $A$ ", satisfaz todas as condicionais do problema e, embora não seja reivindicada, a possível verdade de " $C$ " é plenamente assegurada.

"No caminho para a barbearia" já não é paradoxal para a lógica contemporânea, dada a atual noção de implicação, onde a formalização do problema sob a notação do Cálculo Proposicional Clássico, a saber, "( $C \rightarrow(A \rightarrow$ $\neg B)) \wedge(A \rightarrow B)$ ", consiste em uma fórmula contingente, verdadeira nos casos em que " $C$ " é verdadeira e "A" é falsa. Inclusive Bertrand Russell (1872-1970) cita o quebra-cabeças carrolliano em uma nota de rodapé da obra "The

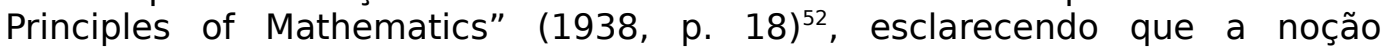
verofuncional que adota para os condicionais lógicos dissolve o problema na medida em que não só permite, mas exige que "A implique $B$ " e "A implique não-B" sejam simultaneamente verdadeiras quando " $\mathrm{A}$ " é falsa.

Assim, nos mesmos moldes da noção verofuncional adotada pelos lógicos contemporâneos, a interpretação semântica proposta por Carroll satisfaz todas as condicionais do problema, onde a implicação " $(A \rightarrow \neg B)$ " é

${ }^{52}$ RUSSELL, B. The Principles of Mathematics. Abingdon, Inglaterra: Routledge Classics, 2010, p. 18. 
verdadeira tendo o antecedente e o consequente falsos, a implicação “ $\mathrm{C} \rightarrow(\mathrm{A}$ $\rightarrow \neg B)$ " é verdadeira independente do valor de verdade do antecedente tendo o consequente verdadeiro, e a implicação " $(A \rightarrow B)$ " é verdadeira graças à falsidade do antecedente.

Embora o exemplo de Carroll colida com a noção verofuncional de implicação adotada pelos lógicos contemporâneos, ele não aceita explicitamente que " $(A \rightarrow \neg B)$ " e " $(A \rightarrow B)$ " seja derivável de todos os casos nos quais " $A$ " é falsa (como o faz Bertrand Russell), tendo defendido apenas um exemplo específico de tal consistência, cuidadosamente ancorado sobre um caso no qual " $A$ " é falsa, mas sua hipótese de verdade é compatível com " $B$ " e " $\neg B$ ". Apesar de não ter defendido a concepção verofuncional da noção de implicação adotada pelos lógicos contemporâneos stricto sensu, seu exemplo contempla todos os casos de valores de verdade do antecedente e consequente nos quais a implicação pode ser verdadeira, estando em plena sintonia com a noção contemporânea.

\section{Considerações finais}

Dada a apresentação do método por subscritos carrolliano, propedêutica à análise dos paradoxos, percebe-se que, diferente de Frege (1879), que parte do cálculo proposicional e acrescenta uma teoria da quantificação, isto é, "um método para simbolizar e exibir rigorosamente as inferências cuja validade depende de expressões como 'todos' ou 'alguns', 'qualquer' ou 'cada um', 'nada' ou 'nenhum'" (KENNY, 1999, p. 438) ${ }^{53}$, Carroll prioriza a lógica silogística na criação de seu método por subscritos, apenas acrescentando a possibilidade de uma leitura proposicional à mesma notação, plasticidade comum aos lógicos do período. Priorizar a silogística em detrimento da lógica proposicional é um reflexo de seu tempo, onde, apesar dos avanços dos algebristas, o legado da lógica aristotélica ainda era muito influente.

A análise do primeiro paradoxo, chamado "Pseudomenos", sugere que Carroll, com uma abordagem mais simples, chegou à mesma solução derivada da teoria de hierarquias entre linguagens e metalinguagens de Tarski, a saber, que a proposição "Eu estou dizendo uma mentira" não pode, necessariamente, referir-se a si mesma como seu próprio assunto.

A análise do segundo paradoxo, chamado "Antistrephon", apresenta uma possível formalização para a solução carrolliana, segundo a qual o paradoxo consiste em um excesso de regras. Percebe-se que a solução de Leibniz para tal paradoxo parte da suposição de uma das possibilidades levantadas por Carroll.

Por sua vez, o exame do terceiro paradoxo, chamado "On the Way to the Barber-shop", único paradoxo original analisado, levou ao reconhecimento de uma importante contribuição carrolliana para o desenvolvimento da noção lógica de implicação, sendo o diagnóstico de um problema central para a lógica simbólica, onde Carroll parece antecipar a noção que veio a ser adotada pelos lógicos contemporâneos.

O presente trabalho limitou-se ao exame de três dos treze paradoxos que receberam tratamento lógico de Lewis Carroll na obra "Symbolic Logic" (1986, p. 423-475)54. Dada a importância que os paradoxos desempenharam no período, a continuação lógica deste trabalho consiste na análise dos demais paradoxos, a fim de esclarecer os pormenores do desenvolvimento

\footnotetext{
${ }^{53}$ KENNY, A. História Concisa da Filosofia Ocidental. Tradução de Desidério Murcho,Fernando Martinho, Maria José Figueiredo, Pedro Santos e Rui Cabral. Lisboa: Temas e Debates Actividades Editoriais, 1999, p. 438.

${ }^{54}$ CARROLL, L. Symbolic Logic: Lewis Carroll's. 6 ed. Rev., ampl. e atual. New York: Clarkson Potter, $1986,514 \mathrm{p}$. 
lógico que estava ocorrendo no período e conhecer as importantes marcas deixadas por Lewis Carroll na história da lógica.

\section{Referências}

ARTOSI, A; PIERI, B; SARTOR, G. (Eds.). Leibniz: Logico-Philosophical Puzzles in the Law: Philosophical Questions and Perplexing Cases in the Law. New York: Springer, 2013. p. xx-xxii.

BARTLEY III. W. W. Annotations. In: CARROLL, L. Symbolic Logic: Lewis Carroll's. 6. ed. Rev., ampl. e atual. New York: Clarkson Potter, 1986. 514 p.

BEALL, J; GLANZBERG. M; RIPLEY, D. Liar Paradox. In: ZALTA, E. Z. et al (Org.). Stanford Encyclopedia of Philosophy. Stanford, California. 12 Dez. 2016. Disponível em: <https://plato.stanford.edu/entries/liarparadox/\#TarsHierLang>. Acesso em: 3 set, 2017.

BOOLE, G. An Investigation of the Laws of Thought: on which are founded: TheMathematical Theories of Logic and Probabilities. Cambridge: McMillan and Co., 1854. 425 p.

CARROLL, L. Alice: edição comentada. Introdução e Notas: Martin Gardner. Trad. Maria Luiza Borges. Rio de Janeiro: Jorge Zahar Ed., 2002.

CARROLL, L. Symbolic Logic: Part 1 -Elementary. Londres: MacMillan and Co., 1896.

$188 \mathrm{p.}$

CARROLL, L. Symbolic Logic: Lewis Carroll's. 6 ed. Rev., ampl. e atual. New York:

Clarkson Potter, 1986. 514 p.

CARROLL, L. The Game of Logic. 2 ed. London: MacMillan and Co., 1887.

FREGE, G. Begriffsschrift: eine der arithmetischen nachgebildete Formelsprache des reinen Denkens. Halle, Alemanha: Louis Nebert, 1879. 89 p.

GABBAY, D.M.; GUENTHNER, F. (Eds.). Handbook of Philosophical Logic: Volume 6. 2 ed. New York: Springer, 2002. p. 12.

KENNY, A. História Concisa da Filosofia Ocidental. Tradução de Desidério Murcho, Fernando Martinho, Maria José Figueiredo, Pedro Santos e Rui Cabral. Lisboa: Temas e Debates - Actividades Editoriais, 1999. p. 438.

LINDEMANN, J. L. A Lógica de Lewis Carroll. Dissertação (mestrado) Universidade Federal de Santa Maria, Centro de Ciências Sociais e Humanas, Programa de Pós-Graduação em Filosofia, RS, 2017. 121 p.

McGRATH, M. Propositions. In: ZALTA, E. Z. et al (Org.). Stanford Encyclopedia of Philosophy. Stanford, California. 20 Jun. 2012. Disponível em: <http://plato.stanford.edu/entries/propositions/>. Acesso em: 28 ago, 2017.

MONTOITO, R. Chá com Lewis Carroll: a matemática por trás da literatura. Jundiaí: Paco Editorial, 2011.

OHRSTROM, P.; HASLE, P. F. V. Temporal Logic: From Ancient Ideas to Artificial Intelligence. Dordrecht, Holanda: Kluwer Academic Publishers, 1995. p. 19-20.

RUSSELL, B. The Principles of Mathematics. Abingdon, Inglaterra: Routledge Classics, 2010. p. 18. 
SAUTTER, F. Lewis Carroll e a pré-história das árvores de refutação. In. SAUTTER, F. T. \& FEITOSA, H. de A. (Eds.). Lógica: teoria e reflexões. Coleção CLE. Campinas, 2004.

SMULLYAN, R. M. Languages in Which Self Reference is Possible. In: The Journal of Symbolic Logic. Cambridge, v. 22, n. 1, p. 55-67. mar. 1957.

THE JOY OF LOGIC. Direção: Catherine Gale. 59 min, [S.I.]: BBC, 2013. Disponível em <http://www.dailymotion.com/video/xla6ogd_the-joy-oflogic_tech>. Acesso em: 03 set. 2017.

Doutorando em Filosofia (UFSM) Email: johnllindemann@hotmail.com

Doutor em Filosofia (UNICAMP, 2000) Professor Titular da UFSM E-mail: ftsautter@ufsm.br 